\title{
Calcium-binding protein S100A4 confers mesenchymal progenitor cell fibrogenicity in idiopathic pulmonary fibrosis
}

\author{
Hong Xia, ${ }^{1}$ Adam Gilbertsen, ${ }^{1}$ Jeremy Herrera, ${ }^{1}$ Emilian Racila, ${ }^{2}$ Karen Smith, ${ }^{1}$ Mark Peterson, ${ }^{1}$ Timothy Griffin, ${ }^{3}$ \\ Alexey Benyumov, ${ }^{1}$ Libang Yang, ${ }^{1}$ Peter B. Bitterman, ${ }^{1}$ and Craig A. Henke ${ }^{1}$ \\ 'Departments of Medicine, ${ }^{2}$ Laboratory Medicine \& Pathology, ${ }^{3}$ Biochemistry, Molecular Biology, and Biophysics, University of Minnesota, Minneapolis, Minnesota, USA.
}

\begin{abstract}
Idiopathic pulmonary fibrosis (IPF) is a progressive disease with a prevalence of 1 million persons worldwide. The fibrosis spreads from affected alveoli into contiguous alveoli and leads to death by asphyxiation. We previously discovered that the IPF lung harbors fibrogenic mesenchymal progenitor cells (MPCs) that serve as a cell of origin for disease-mediating myofibroblasts. In a prior genomewide transcriptional analysis, we found that IPF MPCs displayed increased expression of S100 calcium-binding A4 (S100A4), a protein linked to cancer cell proliferation and invasiveness. Here, we have examined whether S100A4 mediates MPC fibrogenicity. Ex vivo analysis revealed that IPF MPCs had increased levels of nuclear S100A4, which interacts with L-isoaspartyl methyltransferase to promote $\mathrm{p} 53$ degradation and MPC self-renewal. In vivo, injection of human IPF MPCs converted a self-limited bleomycin-induced mouse model of lung fibrosis to a model of persistent fibrosis in an S100A4-dependent manner. S100A4 gain of function was sufficient to confer fibrotic properties to non-IPF MPCs. In IPF tissue, fibroblastic foci contained cells expressing Ki67 and the MPC markers SSEA4 and S100A4. The expression colocalized in an interface region between myofibroblasts in the focus core and normal alveolar structures, defining this region as an active fibrotic front. Our findings indicate that IPF MPCs are intrinsically fibrogenic and that S100A4 confers MPCs with fibrogenicity.
\end{abstract}

\section{Introduction}

Idiopathic pulmonary fibrosis (IPF) is a progressive fibrotic lung disease characterized by the proliferation of fibroblasts that deposit collagen within alveolar structures, forming the signature morphologic lesion of IPF - the fibroblastic focus (1-8). IPF progresses in a stereotypic fashion, expanding centrally from the peripheral basilar portions of the lung. Fibroblastic foci are frequently located at the boundary between relatively normal alveolar structures and regions of fibrosis. They are heterogeneous structures that vary greatly in size and shape, ranging from small discrete lesions to large serpiginous structures $(9,10)$. While the reason for the variation in size and shape are unclear, one possible explanation is that there is temporal growth of fibroblastic foci as the fibrotic lesions evolve due to the spread of fibrosis into adjacent normal alveolar structures. Our prior studies revealed a distinct IPF fibroblast phenotype characterized by aberrant integrin signaling, leading to pathological activation of proliferation and survival pathways (11-13); they also revealed that IPF lung harbors pathologic mesenchymal progenitor cells (MPCs) that are cells of origin for IPF fibroblasts (14). However, the mechanism(s) underlying IPF MPC fibrogenicity and their role in the stereotypic progression of fibrosis are not known.

Gene expression profiling of IPF MPCs distinguished them from MPCs isolated in a similar manner from the lungs of patient

Conflict of interest: The authors have declared that no conflict of interest exists. Submitted: September 21, 2016; Accepted: April 6, 2017.

Reference information: / Clin Invest. 2017;127(7):2586-2597.

https://doi.org/10.1172/JCI90832. controls, with enrichment of genes associated with diseaserelevant ontologies (14). Specifically, we found that IPF MPCs express higher levels of S100A4 compared with control MPCs (14). S100A4, a calcium binding protein, is upregulated in cancer stem cells, where it controls proliferation, differentiation, and metastasis (15-22). S100A4 also amplifies TGF- $\beta$-induced fibroblast activation in the progressive fibrotic disorder systemic sclerosis (23). S100A4 has nuclear, cytoplasmic, and extracellular functions (24). S100A4 regulates cellular function through its interaction with other proteins. In the nucleus, S100A4 interacts with p53 protein, triggering its degradation by the proteasome, which promotes cell proliferation; refs. 25-28). Notably, S100A4-p53 interaction in cardiac fibroblasts has been liked to cardiac fibrosis (29). Within the cytoplasm, S100A4 binds directly with nonmuscle myosin IIA, regulating cell motility/invasion (30). Reports indicate that secreted, extracellular S100A4 modulates the differentiated state of neighboring cells via damage-associated molecular pattern signaling (31-33). Thus, depending on its location, S100A4 is capable of regulating a number of cellular processes crucial to fibrotic progression. Therefore, we analyzed the role S100A4 might play in regulating IPF MPC fibrogenicity and fibrotic progression.

\section{Results}

IPF MPCs display increased S10OA4, which localizes to the nucleus. We previously discovered that IPF lung tissue harbors pathologic MPCs that are cells of origin for IPF fibroblasts (14); however, the molecular mechanisms underlying their fibrogenicity remain undefined. To identify molecular processes that might impart IPF 
A

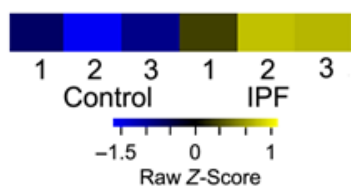

B

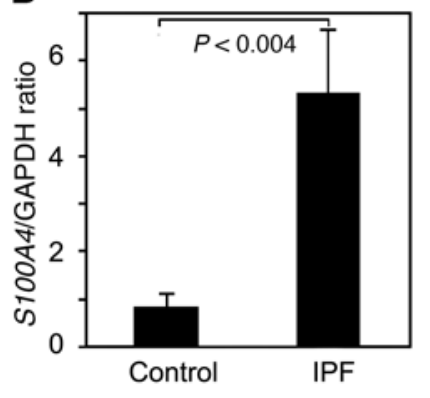

C

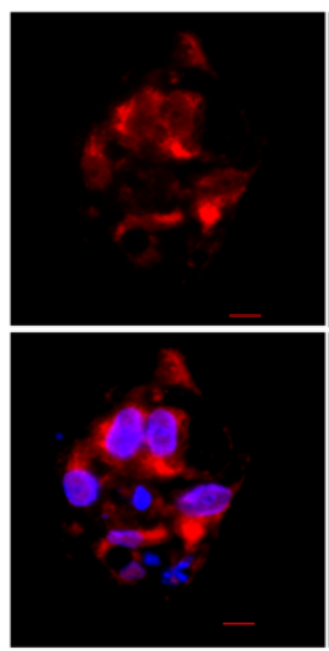

Control
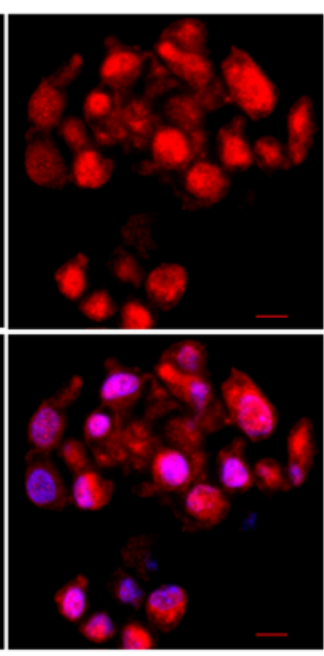

IPF
D

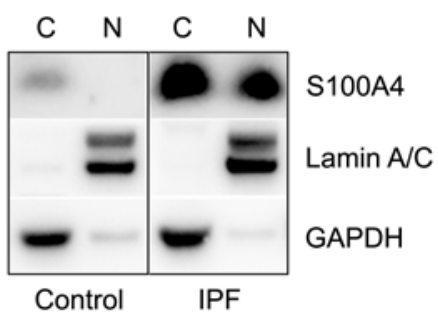

Figure 1. IPF MPCs display increased S100A4, which localizes to the nucleus. (A) Genome-wide RNA sequencing data. Shown is the heatmap for S100A4 gene expression in IPF and control MPCs derived from 3 IPF patients and 3 control patients based on our previously published data (14). Colors represent per gene $Z$ score (expression difference normalized for standard deviation). (B) Validation testing for S100A4 mRNA expression in IPF and control MPCs. Shown are relative expression levels of each mRNA by qPCR. Data shown represents the mean mRNA levels in IPF MPCs derived from 3 cell lines. (C) Immunocytochemical analysis illustrating S100A4 location in IPF and control colony-forming MPCs (red, S100A4; blue, DAPI; $n$ = 3). Scale bar: $5 \mu$ m. (D) Western blot analysis of S100A4 expression in nuclear (N) and cytoplasmic (C) fractions of IPF and control MPCs. Lamin A/C and GAPDH were used as loading controls. Data are expressed as mean \pm SEM. $P$ value in B was determined by 2 -tailed Student's $t$ test.

MPCs with their pathological properties, we reexamined our transcriptomic comparison of IPF MPCs and controls. Among the genes more highly expressed in IPF MPCs, we found S100A4 (Figure 1A), also termed metastasin. S100A4 is linked to cancer cell proliferation and invasion (15) and is highly expressed in cancer stem cells, where it is located in the nucleus (16-19). To test the validity of our transcriptional profiling, we quantified S1OOA4 mRNA and confirmed increased expression in IPF MPCs (Figure 1B). Immunocytochemical analysis of IPF MPCs demonstrated S100A4 in both the nucleus and cytoplasm, with high levels within the nucleus, whereas - in control MPCs - S100A4 was predominately located in the cytoplasm (Figure 1C). Immunoblot analysis of nuclear and cytoplasmic fractions confirmed that IPF MPCs contain higher S100A4 protein levels in the nucleus compared with control (Figure 1D and Supplemental Figure 1; supplemental material available online with this article; https://doi.org/10.1172/JCI90832DS1).

Characterization of the IPF MPC S100A4 nuclear interactome. Prior studies have shown that S100A4 interacts with other proteins to regulate cell function $(25,26,29)$. To elucidate the mechanism(s) by which nuclear S100A4 might regulate IPF MPC function, we first defined the S100A4 nuclear interactome by isolating the IPF MPC nuclear fraction, followed by I of S100A4 using an anti-S100A4 antibody. The negative control consisted of immunoprecipitation using a nonspecific IgG antibody. The resulting nuclear lysate was subjected to SDS-PAGE, followed by silver staining. Bands were excised and subjected to in-gel digestion by trypsin and protein identification. Peptide sequences were identified by tandem mass spectrometry and sequence database searching, followed by inference of protein identities based on the peptide sequences. Analysis using the Peaks Studio 7.0 build 20140912 (Bioinformatics Solutions) software package revealed
S100A4 interaction with proteins known to regulate p53. This included protein-L-isoaspartyl methyltransferase (PIMT, also known as PCMT1) and multiple members of the proteasome complex (Supplemental Table 1).

S1OOA4 promotes p53 degradation and IPF MPC self-renewal. To determine whether S100A4 regulates p53 expression, we performed gain- and loss-of-function studies. Overexpression of S100A4 in IPF MPCs decreased p53 expression, whereas knockdown of S100A4 by shRNA augmented p53 expression (Figure 2A). Similar results were seen using control MPCs (Supplemental Figure 2A). p53 binds the $P 21$ promoter, increasing p21 expression and, thus, inhibiting cell cycle progression. Therefore, we examined the effect of gain or loss of S100A4 on p21 expression. Consistent with the p53 results, gain of S100A4 decreased p21 levels, whereas loss of S100A4 increased p21 expression (Figure 2A). These data show the importance of S100A4 in control of cell cycle checkpoint regulators in lung MPCs.

Prior work has implicated S100A4 in cancer stem cell selfrenewal (15-17). Since S100A4 interacts with proteins regulating cell cycle transit in IPF MPCs, we examined its role on IPF MPC self-renewal in a colony-forming assay. Overexpression of S100A4 increased IPF MPC colony number by 2.3-fold and colony size by 1.6-fold compared with control, whereas knockdown of S100A4 reduced IPF MPC colony number by $89 \%$ and colony size by $53 \%$ compared with control (Figure 2B). Similarly, S100A4 gain of function in control MPCs augmented colony formation, while S100A4 loss of function diminished control MPC colony formation (Supplemental Figure 2B). Based on our finding that S100A4 loss of function increases p53 and p21 levels, and since high levels of p53 and p21 are associated with cell cycle arrest and senescence, we examined the effect of S100A4 knockdown on $\beta$-galactosidase 


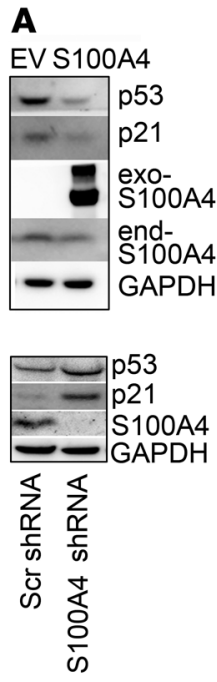

B
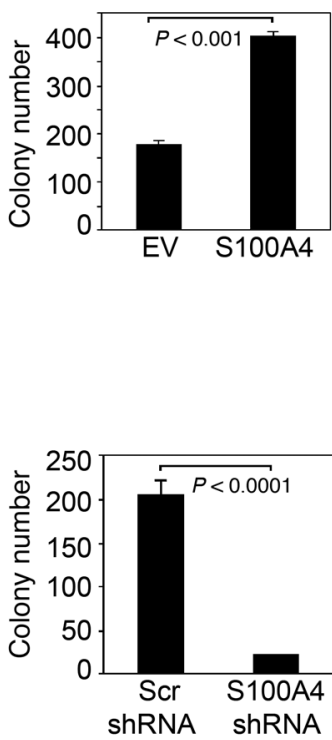
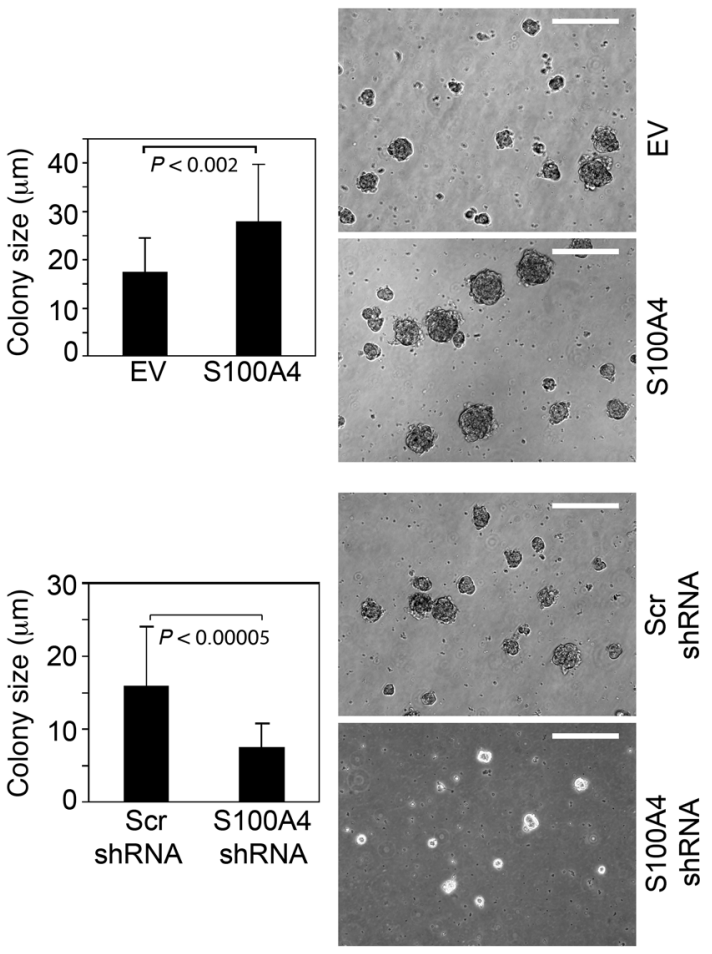

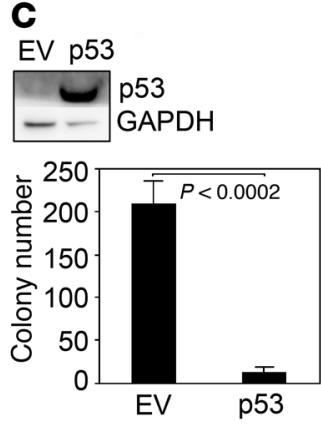

D

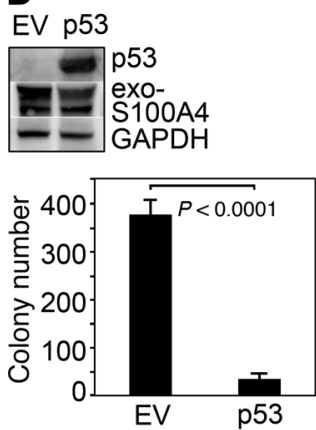

Figure 2. S100A4 regulates IPF MPC self-renewal. (A) IPF MPCs were infected with lentiviral vector containing WT S100A4 (S100A4) or empty vector (EV; top panel) or S100A4 shRNA or scrambled (Scr) shRNA (bottom panel) to achieve gain or loss of function. p53, p21, and S100A4 expression were quantified by Western blot analysis. GAPDH was used as loading control. Exo-S100A4, exogenous S100A4; end-S100A4, endogenous S100A4. (B) IPF MPCs were infected with a lentiviral vector containing WT S100A4 (S100A4) or empty vector control (EV) or S100A4 shRNA or scrambled (Scr) shRNA. Five-thousand cells were seeded per well in 24-well dishes containing methylcellulose, and they were cultured for 7 days $(n=3)$. Left panels: colony number was quantified by enumeration. Middle and right panels: graphs depicting colony size and phase contrast image of IPF MPC colony formation, respectively. Scale bar: $50 \mu \mathrm{m}$. (C) IPF MPCs were infected with an adenoviral vector containing WT p53 (p53) or empty vector (EV) and cultured in methylcellulose for 7 days. Left panel: Western blot analysis was performed to assess $\mathrm{p} 53$ protein expression. Right panel: colony number was quantified by enumeration $(n=3)$. (D) IPF MPCs overexpressing S100A4 were infected with an adenoviral vector containing WT p53 (p53) or empty vector control (EV). Left panel: Western blot analysis was performed to assess $\mathrm{p} 53$ protein expression. GAPDH was used as a loading control. Right panel: colony number was quantified by enumeration $(n=3)$. The data were replicated using MPCs isolated from an independent primary mesenchymal cell line. Data are expressed as mean \pm SEM. $P$ values in B-D were determined by 2-tailed Student's $t$ test.

expression. Knockdown of S100A4 in IPF MPCs led to increased $\beta$-galactosidase expression (Supplemental Figure 3). Our data suggest that S100A4 promotes IPF MPC self-renewal by decreasing p53 levels. To analyze the effect of p53 on IPF MPC self-renewal, we overexpressed WT p53 in IPF MPCs. Gain of p53 function increased p53 protein levels and inhibited IPF MPC self-renewal (Figure 2C). We also examined the effect of $\mathrm{p} 53$ gain of function on IPF MPC self-renewal in IPF MPCs overexpressing S100A4. p53 gain of function elevated p53 levels, and IPF MPC self-renewal was suppressed, even in the context of excess S100A4 (Figure 2D). These data suggest that high nuclear S100A4 levels function to promote IPF MPC self-renewal and population expansion by lowering p53/p21 levels, enabling the cells to escape cell cycle arrest and senescence.

S100A4 interacts with p53, promoting its degradation by the proteasome (25-28). Since we found that S100A4 complexed with members of the proteasome, we examined whether S100A4 promotes IPF MPC self-renewal by stimulating $\mathrm{p} 53$ proteasomal degradation. We treated IPF MPCs with nutlin-3, which disrupts the interaction of p53 with HDM2, the ubiquitin E3 ligase responsible for p53 ubiquitination. Nutlin-3 augmented p53 and p21 expression in IPF MPCs compared with cells treated with vehicle control (Figure 3A). We also examined the effect of the proteasome inhibitor MG-132 on p53 and p21 expression. Similar to the effect of nutlin-3, IPF MPCs treated with MG-132 had higher p53 and p21 protein levels compared with vehicle control (Figure 3B). We next treated IPF MPCs overexpressing S100A4 with nutlin-3 and examined its effect on 53 protein levels. Nutlin-3 treatment stabilized p53 in IPF MPCs overexpressing S100A4 compared with control (Figure 3C). These data are consistent with the concept that, in IPF MPCs, S100A4 decreases p53 protein expression by promoting its proteasomal degradation.

Our mass spectrometry studies identified PIMT as a member of the S100A4 nuclear interactome. PIMT destabilizes p53 by enhancing p53-HDM2 interaction. PIMT is a methyltransferase, and its effects on p53 stability occur via PIMT-mediated methylation of p53 (34). Immunoprecipitation studies demonstrated that S100A4 exists in a protein complex with PIMT in IPF MPCs, confirming the mass spectrometry results (Figure 3D). To determine whether S100A4 regulates IPF MPC self-renewal via PIMT, 
A

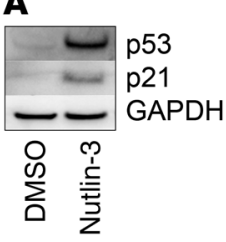

$\mathbf{E}$

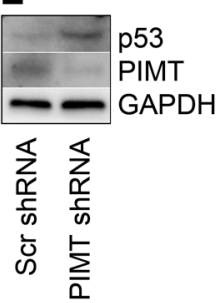

B
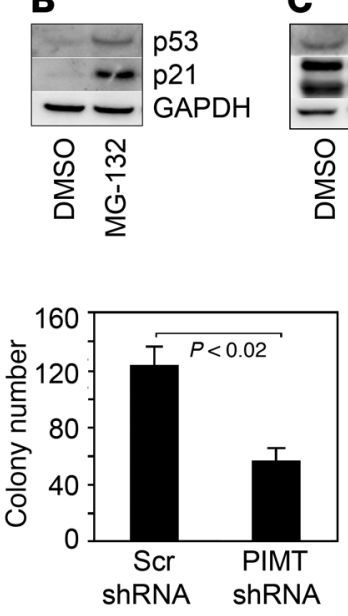

C

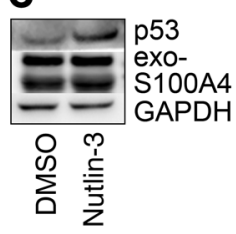

D

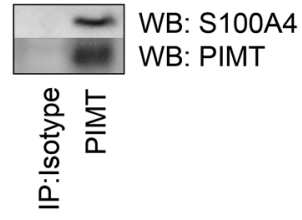

Figure 3. S100A4 promotes $\mathrm{p} 53$ degradation via the proteasome and its interaction with PIMT. (A and B) IPF MPCs were treated with $10 \mu \mathrm{M}$ nutlin-3, $5 \mu \mathrm{M}$ MG-132, or DMSO control for 4 hours. The cells were harvested, and Western blot analysis was performed to quantify p53 and p21 levels. GAPDH was used as a loading control. (C) IPF MPCs overexpressing WT S100A4 were treated with $10 \mu \mathrm{M}$ nutlin-3 (DMSO served as control) for 4 hours, and Western blot analyses for $\mathrm{p} 53$ and S1000A4 expression were performed. (D) PIMT was immunoprecipitated (IP) from IPF MPC cell lysates and Western blot (WB) analysis for S100A4 and PIMT was performed. Lane 1: IP using isotype control antibody. Lane 2: IP using PIMT antibody. (E) PIMT was knocked down in IPF MPCs using PIMT shRNA. Scrambled shRNA served as control. The cells were placed in methylcellulose and cultured for 7 days. Left panel: $p 53$ and PIMT expression were quantified by Western blot analysis. Right panel: colony number was quantified by enumeration $(n=3)$. (F) S100A4 was first overexpressed in IPF MPCs using a lentiviral vector containing a WT S100A4 construct. PIMT was then knocked down using PIMT shRNA. Cells treated with scrambled shRNA served as control. The cells were placed in methylcellulose and cultured for 7 days. Left panel: p53, PIMT, and S100A4 expression were quantified by Western blot analysis. Right panel: colony number was quantified by enumeration $(n=3)$. The data were replicated using MPCs isolated from an independent primary mesenchymal cell line. Data are expressed as mean \pm SEM. $P$ values in $\mathbf{E}$ and $\mathbf{F}$ were determined by 2-tailed Student's $t$ test.

this, we found that mice treated with low-dose intratracheal bleomycin $(1.25 \mathrm{U} / \mathrm{kg})$ displayed increased collagen content 2 weeks after bleomycin administration compared with baseline. However, by week 6, collagen content had declined back to the baseline of untreated mice (Figure 4B), and histological analysis of lungs at week 6 showed only scant areas of collagen deposition and were devoid of human cells expressing procollagen I (Supplemental Figure 4).

To determine whether IPF MPCs can drive fibrosis, we examined the ability of human IPF MPCs to convert the bleomycin model of fibrosis from a self-limited regressing model to a model of persistent fibrosis. To do this, we first treated mice with low-dose intratracheal bleomycin to establish mice with self-limited lung fibrosis. Two weeks after bleomycin treatment, during the peak time frame of the fibrotic response (36), we injected IPF or control MPCs via the tail vein (Figure 4A). Four weeks following MPC administration, the lungs were removed and analyzed for fibrosis. The lungs of mice receiving IPF MPCs had $\sim 5$-fold increased collagen content compared with mice receiving

we knocked down PIMT in IPF MPCs and examined p53 protein expression and colony formation. Knockdown of PIMT stabilized p53 levels and decreased IPF MPC colony formation by $54 \%$ (Figure $3 \mathrm{E}$ ). Since overexpression of S100A 4 decreased $\mathrm{p} 53$ expression while increasing IPF MPC colony formation, we next determined whether this effect was mediated through PIMT. We knocked down PIMT in IPF MPCs overexpressing S100A4. Even in the context of increased S100A4, PIMT depletion augmented p53 protein levels while inhibiting IPF MPC colony formation by $40 \%$ (Figure $3 \mathrm{~F}$ ), demonstrating a pivotal role for PIMT in the ability of S100A4 to promote $\mathrm{p} 53$ degradation. These results indicate that $\mathrm{S} 100 \mathrm{~A} 4 \mathrm{com}-$ plexes with PIMT and members of the proteasome, promoting degradation of $\mathrm{p} 53$, loss of $\mathrm{p} 21$ expression, and IPF MPC self-renewal.

Human IPF MPCs convert the mouse bleomycin model of lung fibrosis from a self-limited, regressing model to a model of persistent fibrosis. IPF MPCs are cells of origin for fibrosis-mediating IPF fibroblasts. We have previously shown that the progeny of IPF MPCs form fibrotic lesions when administered to immunedeficient mice, suggesting that IPF MPCs play an integral role in fibrotic progression (14). Here, we sought to determine whether IPF MPCs can drive fibrosis using the mouse bleomycin model of lung fibrosis (Figure 4A). The standard in vivo model of fibrosis using bleomycin is self-limited, with procollagen mRNA expression peaking between weeks 1 and 2 after intratracheal bleomycin administration and fibrosis regressing thereafter $(35,36)$. Consistent with control MPCs $(846 \pm 151 \mu \mathrm{g} /$ left lung vs. $173 \pm 23 \mu \mathrm{g} /$ left lung, $P<$ 0.01 ; Figure $4 \mathrm{~B})$. In contrast, the collagen content of bleomycintreated mice receiving control MPCs did not differ from mice receiving bleomycin only (no cells). Morphological analysis at this time point revealed regions of fibrosis only in mice receiving IPF MPCs (Figure 4, C and D). Importantly, immunohistochemical analysis using an antibody recognizing human procollagen I revealed congruence between regions containing human cells expressing procollagen and regions displaying collagen deposition as determined by Trichrome staining (Figure 4, D and E), proving that the increased fibrosis observed was mediated by human IPF MPCs. Human cells expressing procollagen I formed fibrotic lesions within alveolar walls (Figure 4E, boxed area). Notably, human procollagen I-expressing cells codistributed with S100A4-expressing cells in fibrotic regions (Figure 4, E-J).

Two key features of IPF were observed in the lungs of mice receiving IPF MPCs: i) the presence of peripheral fibrotic lesions containing procollagen $\mathrm{I}^{+}$human cells, and ii) associated cystic lesions reminiscent of honeycomb changes (Figure 4, K-M). Cells in these fibrotic regions also expressed S100A4 (Figure $4 \mathrm{~N}$ and Supplemental Figure 5). Prior work indicates that there is minimal S100A4 expression 4 weeks after low-dose intratracheal bleomycin (37), suggesting that the S100A4 expression observed is likely of human IPF MPC origin. No morphological evidence of fibrosis was observed in mice receiving bleomycin followed by control 

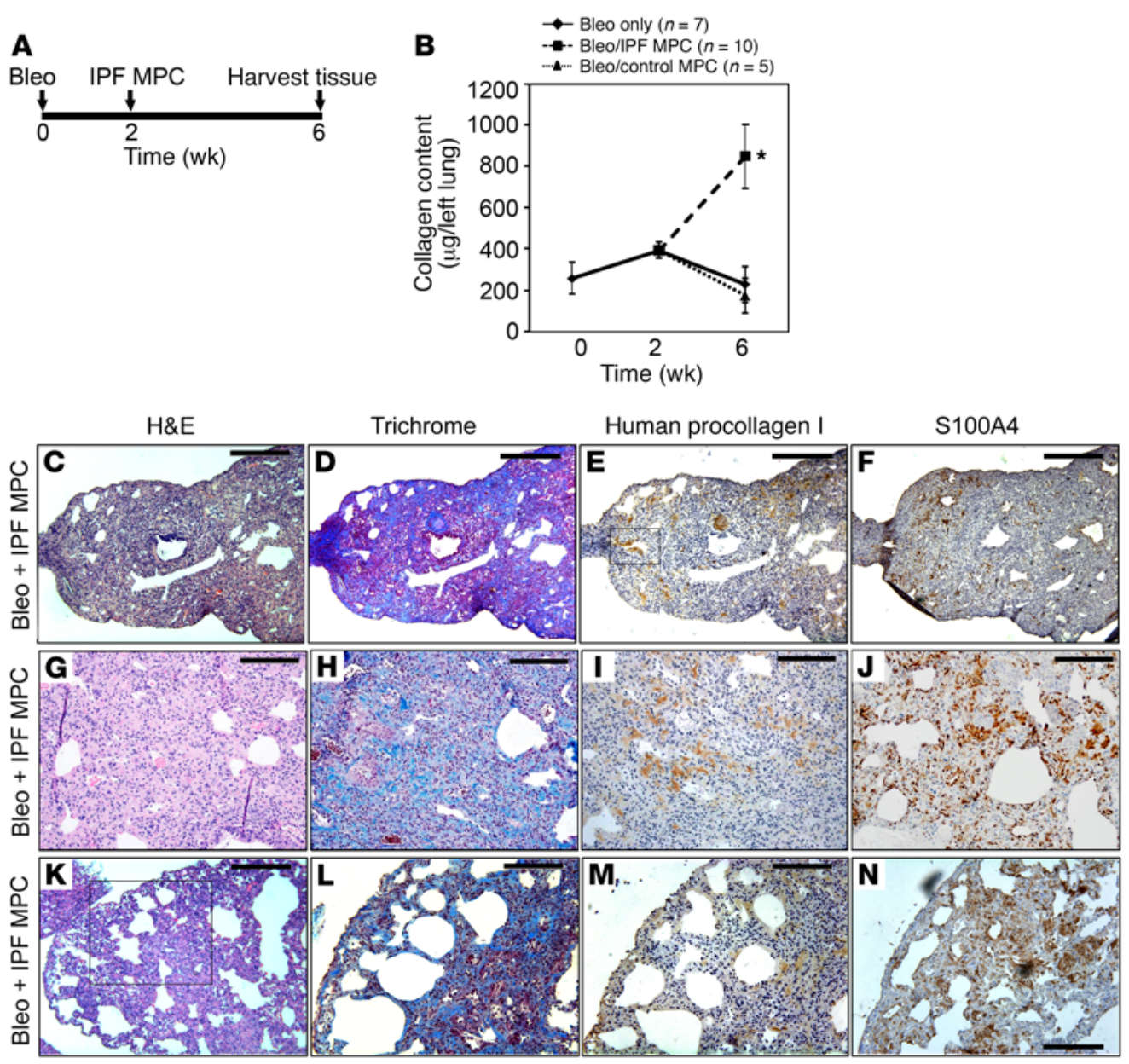

Figure 4. Human IPF MPCs convert the bleomycin model of lung fibrosis from a self-limited model to a model of persistent fibrosis. (A) Schematic displaying timeline of mouse treatment protocol. (B) Three groups of immune-compromised mice were administered intratracheal bleomycin. In one group of mice ("bleo only", $n=7$ ), lungs were harvested at 2 and 6 weeks following bleomycin. In 2 groups of mice, $1 \times 10^{6}$ IPF MPCs ( $n=10$ mice) or control MPCs ( $n=5$ mice) were injected via tail vein 2 weeks after bleomycin. Four weeks after administration of cells, lungs were harvested. Collagen content was quantified in left lungs by Sircol assay (data shown are aggregated from studies done using 2 independent IPF and control MPC lines). Data are expressed as mean \pm SEM. ${ }^{*} P<0.01$, by 2 -tailed Student's $t$ test. (C-N) Serial $4-\mu \mathrm{m}$ sections of right-lung tissue from mice receiving bleomycin followed by IPF MPCs. (C and D) Representative H\&E and Trichrome stains assessing fibrosis and collagen distribution, respectively. Scale bar: $200 \mu \mathrm{m}$. (E and F) IHC using an antibody recognizing human procollagen to identify human cells and assess collagen synthesis (E) and an S100A4 antibody to assess the distribution of S100A4 and human procollagen-expressing cells $(\mathbf{F})$. Boxed area in $\mathbf{E}$ showing procollagen ${ }^{+}$human cells forming a fibrotic lesion obliterating an airspace. Scale bar: $200 \mu \mathrm{m}$. (G-J) Higher-power images demonstrating regions with new collagen deposition (G and $\mathbf{H})$ heavily infiltrated with human cells expressing procollagen and cells expressing S100A4 (I and J). Scale bar: $100 \mu \mathrm{m}$. (K) Morphological analysis demonstrated the presence of peripheral fibrosis with cystic areas. Scale bar: $200 \mu \mathrm{m}$. (L-N) Trichrome stain (L) and IHC (M and $\mathbf{N}$ ) of boxed region in $\mathbf{K}$ showing cystic change with heavy infiltration of procollagen $^{+}$human IPF MPCs codistributed with S100A4-expressing cells in fibrotic lesions. Scale bar: $100 \mu \mathrm{m}$.

human lung MPCs (Supplemental Figure 6). These data demonstrate that IPF MPCs convert the bleomycin model of self-limited regressing lung fibrosis to a model of persistent fibrosis.

S100A4 is necessary and sufficient for MPCs to worsen fibrosis in vivo. Our in vitro studies indicate a key role for S100A4 in regulating IPF MPC self-renewal, while our in vivo studies demonstrate that IPF MPCs worsen fibrosis. We next sought to determine whether S100A4 was necessary for IPF MPCs to worsen fibrosis. For these experiments, immune-deficient mice were treated with low-dose intratracheal bleomycin. Two weeks following bleomycin administration, IPF MPCs infected with a lentiviral vector containing S100A4 shRNA (scrambled shRNA served as control) were injected via the tail vein. Of note, S100A4 shRNA reduced S100A4 expression in IPF MPCs to below the limits of detection
(Figure 2A). Four weeks after cell administration, lungs were harvested and collagen content quantified. Strikingly, knockdown of S100A4 markedly decreased the ability of IPF MPCs to worsen fibrosis. Knockdown of S100A4 decreased collagen content to the level observed in mice receiving only low-dose bleomycin (Figure $5 \mathrm{~A})$. H\&E and trichrome staining demonstrated regions of fibrosis in mice receiving IPF MPCs treated with scrambled shRNA (Figure 5, B and C). Procollagen-expressing human cells, as well as S100A $4^{+}$cells, were present in the fibrotic areas (Figure 5, D and E). In contrast, $\mathrm{H} \& \mathrm{E}$ and trichrome staining revealed only scant areas of fibrosis and collagen deposition in the lungs of mice receiving IPF MPCs in which S100A4 had been knocked down (Figure 5, F and $\mathrm{G})$. Consistent with this, immunohistochemical analysis revealed few human cells expressing procollagen in the S100A4-knockdown 

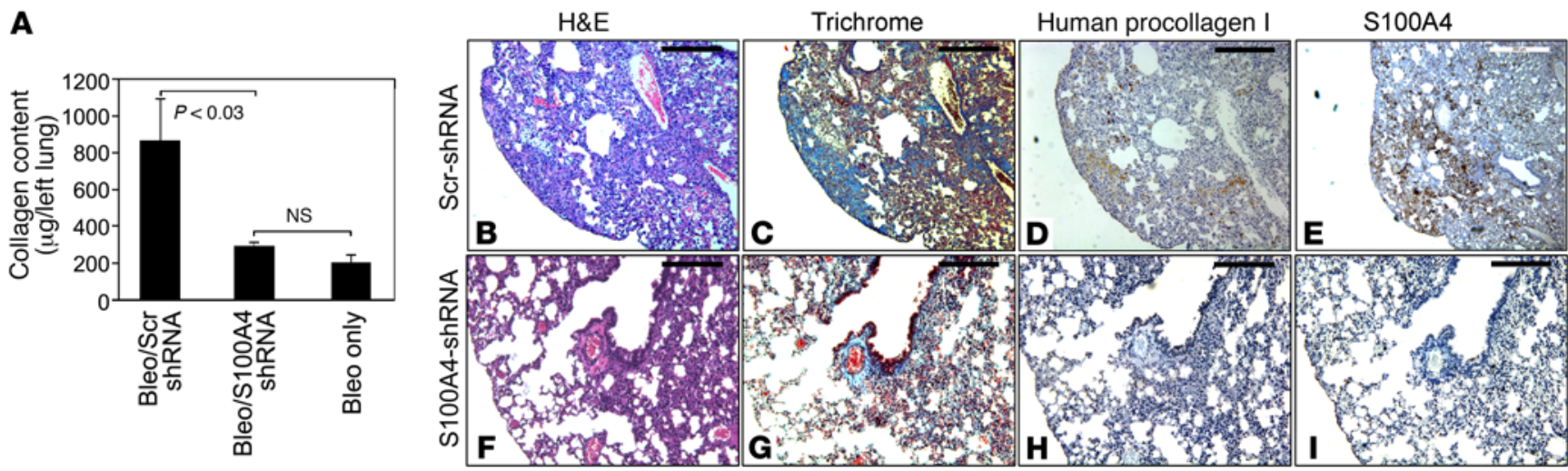

Figure 5. S100A4 is necessary for MPCs to worsen fibrosis in vivo. (A-I) Immune-compromised mice were administered intratracheal bleomycin (1.25 U/kg) to establish lung fibrosis. Two weeks later, IPF MPCs $\left(1 \times 10^{6}\right.$ cells $)$ were infected with a lentiviral vector containing S100A4 shRNA or scrambled shRNA and injected via tail vein into the mice ( $n=5 /$ group). Four weeks after administration of cells, the lungs were harvested. (A) Collagen content in left lungs quantified by Sircol assay. Data are expressed as mean \pm SEM. $P<0.03$ determined by 2-tailed Student's $t$ test. (B-I). Serial 4- $\mu \mathrm{m}$ sections of right-lung tissue from mice receiving IPF MPCs treated with scrambled shRNA (B-E; scale bar: $200 \mu \mathrm{m}$ ) and S100A4 shRNA (F-I; scale bar: $100 \mu \mathrm{m})$. (B, C, F, and G) Shown are representative $\mathrm{H} \& \mathrm{E}$ and trichrome stains to assess fibrosis and collagen distribution in right-lung tissue. (D and $\mathbf{H})$ IHC was performed using an antibody that recognizes human procollagen to identify human cells and assess collagen synthesis. (E and I) IHC was also performed using an S100A4 antibody to assess distribution of S100A4 expression with human procollagen expression.

mice compared with control (Figure 5H) and reduced S100A4 expression (Figure 5I). Of note, the engraftment of human IPF MPCs treated with S100A4 shRNA or scrambled shRNA in the lungs of the immune-deficient mice was confirmed utilizing a realtime PCR method that has been shown to be sensitive for the detection and quantitation of human cells in mice (Supplemental Figure 7; ref. 38). This indicates that - compared with IPF cells in which S100A4 levels were intact - the reduced number of human IPF cells in the mouse lungs, in which S100A4 had been knocked down, cannot be explained by a lack of engraftment. These data indicate that S100A4 is necessary for IPF MPCs to worsen fibrosis.

We next analyzed the effect of S100A4 gain of function in control MPCs on lung fibrosis in the immune-deficient mice pretreated with intratracheal bleomycin. Overexpression of S100A4 in control MPCs increased collagen content by $52 \%$ compared with mice receiving control MPCs treated with empty vector $(288 \pm 18 \mu \mathrm{g} / \mathrm{left}$ lung vs. $190 \pm 12 \mu \mathrm{g} /$ left lung, $P<0.002$; Supplemental Figure $8 \mathrm{~A}$ ). Histological examination of the lungs revealed intravascular and perivascular fibrotic lesions consisting of human cells expressing procollagen that colocalized with S100A4-expressing cells (Supplemental Figure 8B). The lungs of mice receiving control MPCs treated with empty vector showed minimal fibrosis, did not contain intravascular fibrotic lesions, and were largely devoid of human procollagen-expressing cells and S100A4-expressing cells (Supplemental Figure $8 \mathrm{~B}$ ). These data indicate that overexpression of S100A4 is sufficient to confer control MPCs with fibrotic properties.

The IPF fibroblastic focus is a polarized structure containing SSEA4- and S100A4-expressing cells at the periphery of the focus. We previously reported that IPF MPCs, identified by their coexpression of SSEA4 (a marker of stem and progenitor cells) and nuclear OCT4 (a stem cell transcription factor) are found in and around fibroblastic foci in human lung IPF tissue (14). However, as MPCs differentiate, they rapidly lose SSEA4 expression (14), making it difficult to determine the precise number and location of IPF MPCs and their early-generation transit-amplifying daughter cells in fibroblastic foci by IHC using SSEA4 expression as a determinant. Since IPF
MPCs highly express S100A4, we sought to define the location of S100A4-expressing cells in IPF fibroblastic foci. Before performing immunohistochemical studies, we defined S100A4 expression as IPF MPCs differentiating in vitro. SSEA $4{ }^{\text {hi }}$ colony-forming IPF MPCs displayed a gradual reduction in S100A4 expression when cultured on tissue culture plastic dishes coated with type I collagen (Supplemental Figure 9). As they differentiated, they acquired the typical morphologic appearance of myofibroblasts, including $\alpha$-smooth muscle actin ( $\alpha$-SMA) in stress fibers.

We analyzed the location of S100A4-expressing cells in IPF fibroblastic foci in lung pathological specimens from IPF patients $(n=10)$ and patient controls $(n=3)$. We examined fibroblastic foci at the advancing front of fibrosis, where it begins to invade into regions of normal-appearing alveolar structures. We reasoned that, if IPF MPCs are involved in fibrotic progression, they would be found in developing fibrotic lesions as opposed to mature areas of fibrosis, since they rapidly differentiate to myofibroblasts. The data revealed a striking concentration of S100A4-immunoreactive cells within fibroblastic foci (Figure 6A and Supplemental Figure $10 \mathrm{~A})$ primarily localizing to the periphery of the focus, indicating that the fibroblastic focus is a polarized structure. Consistent with our in vitro studies demonstrating S100A4 in a nuclear and cytoplasmic location in IPF MPCs, individual cells with a stromallike morphology could be identified in these regions expressing S100A4 both in the nucleus and cytoplasm (Figure 6B). Scant S100A4 expression was seen in normal alveolar structures in patient control lung tissue specimens (Supplemental Figure 10B). We next compared the location of S100A4-expressing cells with $\alpha$-SMA-expressing cells within the fibroblastic foci. To do this, we performed immunohistochemical analysis of fibroblastic foci double staining for S100A4 and $\alpha$-SMA. Our analysis revealed $\alpha$-SMA ${ }^{+}$ myofibroblasts in the focus core, with S100A4-expressing cells found in an interface region between the focus core and relatively uninvolved contiguous alveolar structures (Figure 6C). Consistent with this, serially sectioned fibroblastic foci revealed $\alpha$-SMA ${ }^{+}$ myofibroblasts expressing procollagen in the focus core (Figure 6, 

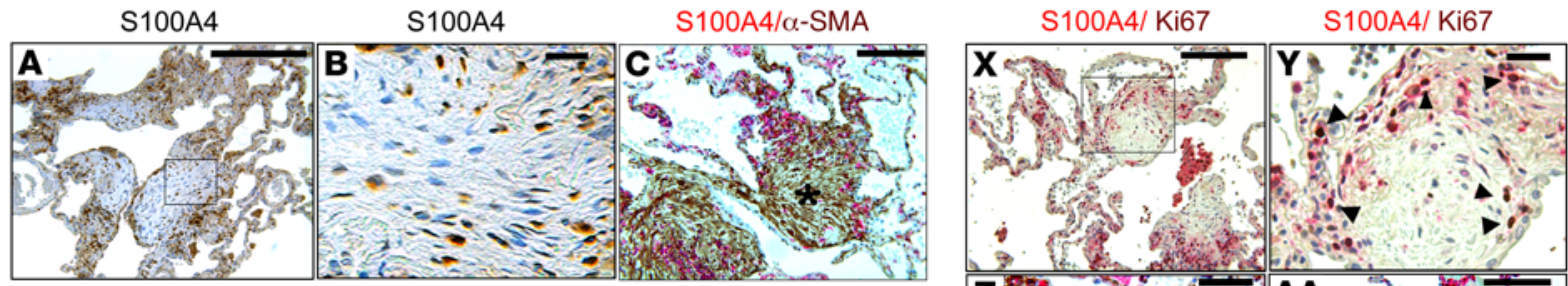

Fibroblastic focus 1 (serial sections)
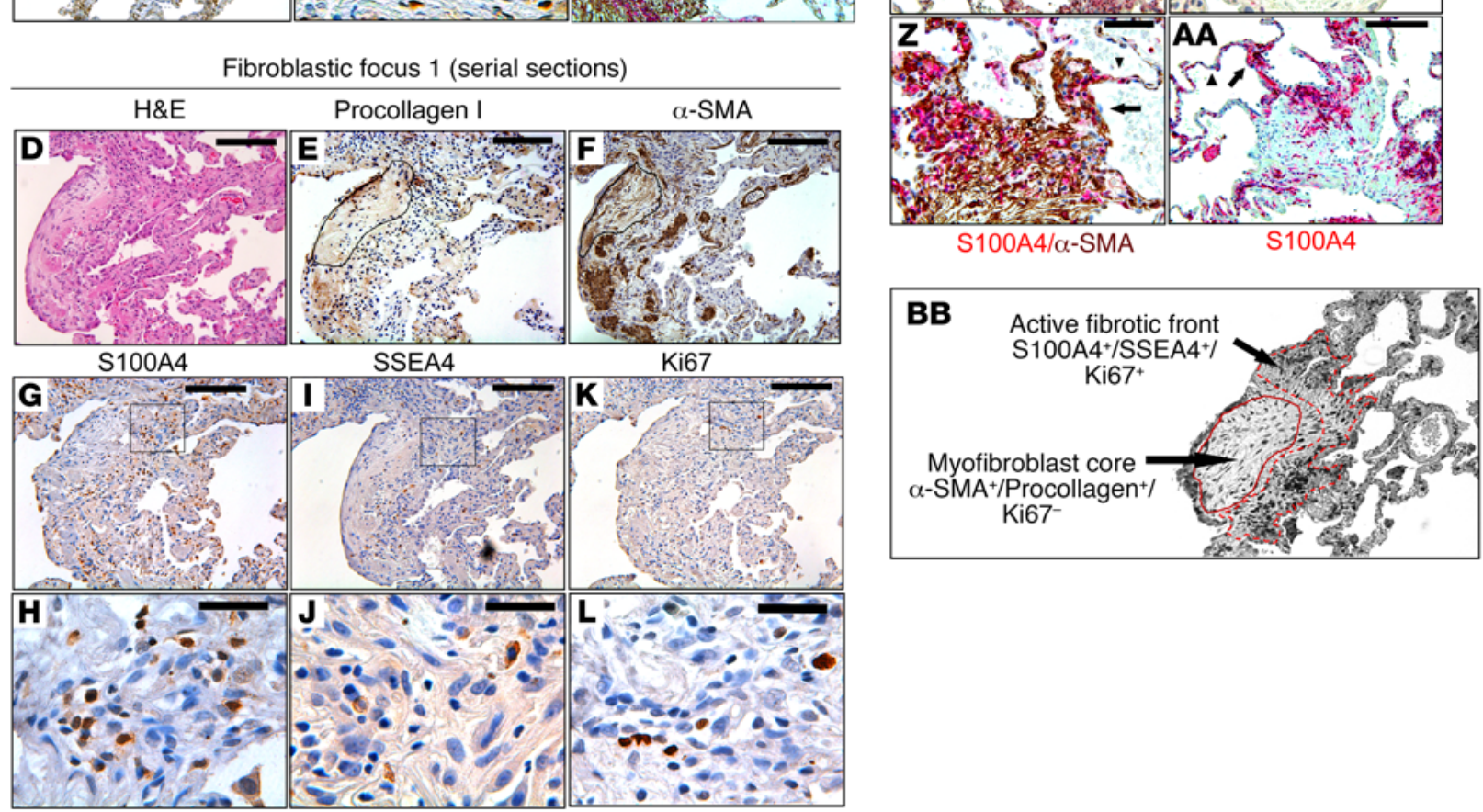

Fibroblastic focus 2 (serial sections)

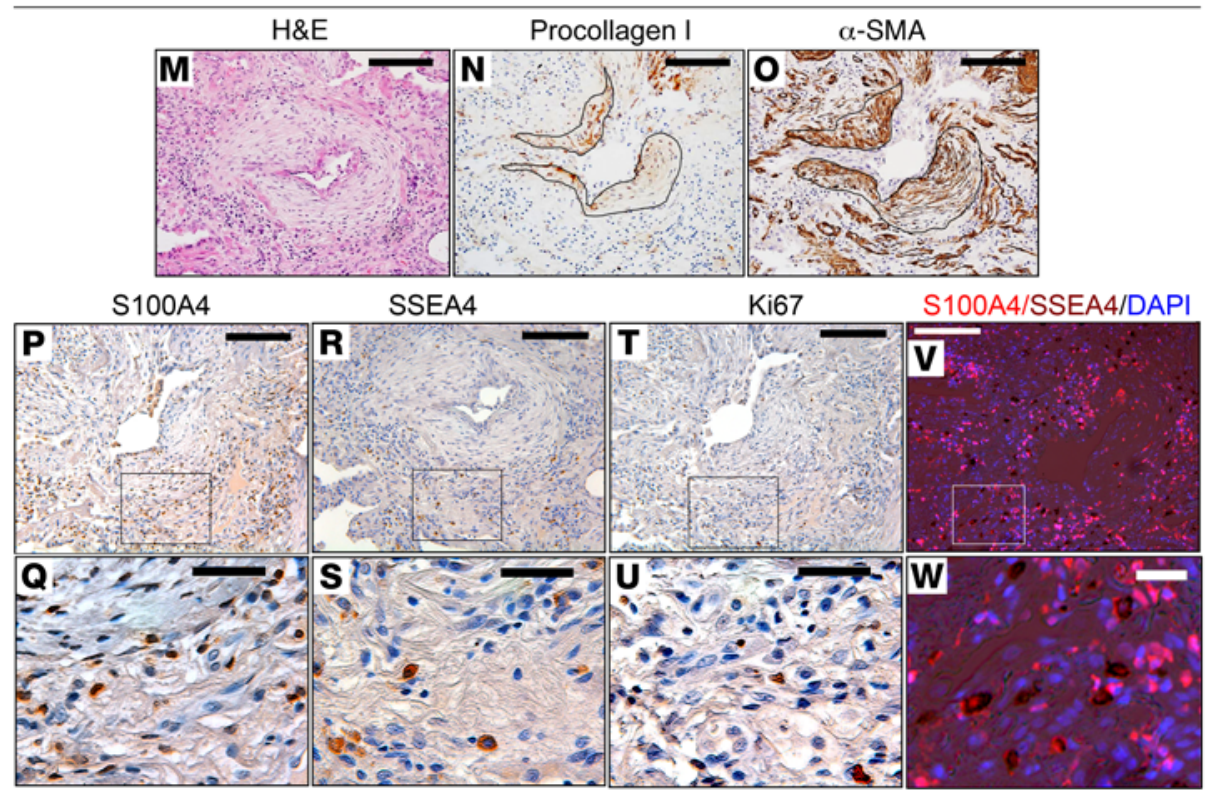


Figure 6. The IPF fibroblastic focus is a polarized structure. IHC was performed on human IPF lung tissue $(n=10)$. (A) IHC showing distribution of S100A4 expression in fibroblastic foci. Scale bar: $200 \mu \mathrm{m}$. (B) Higher-power image of boxed region in A. Scale bar: $20 \mu \mathrm{m}$. (C) Double stain for S100A4 (red) and $\alpha$-SMA (brown) defining the distribution of S100A4- and $\alpha$-SMA-expressing cells in the fibroblastic focus. Asterisk, myofibroblast core. Scale bar: $50 \mu \mathrm{m}$. (D-W) Serial 4- $\mu \mathrm{m}$ sections of 2 representative fibroblastic foci. (D-F and $\mathbf{M}-\mathbf{O}$ ) H\&E (D and $\mathbf{M}$ ), procollagen (E and $\mathbf{N}$ ), and $\alpha-S M A(\mathbf{F}$ and $\mathbf{O}$ ) staining of the focus. Scale bar: $100 \mu \mathrm{m}$. Outline in E, F, N, and $\mathbf{O}$ delineate the focus core. (G-L and $\mathbf{P}-\mathbf{U})$ IHC for S100A4 (G, H, P, and Q), SSEA4 (I, J, R, and S), and Ki67 (K, $\mathbf{L}, \mathbf{T}$, and $\mathbf{U}$ ). (G, I, K, P, R, and T). Scale bar: $100 \mu \mathrm{m}$. (H, J, L, Q, S, and $\mathbf{U})$ Higher-power images of boxed regions in G, I, K, P, R, and T. Scale bar: $20 \mu \mathrm{m}$. (V and W) Low- and high-power images showing IHC for SSEA4 (brown) overlaid with immunofluorescence for S100A4 (red). Nuclei are stained with DAPI. Scale bar: $100 \mu \mathrm{m}(\mathbf{V})$ and $20 \mu \mathrm{m}(\mathbf{W})$. (X) Double stain for S100A4 (red) and Ki67 (nuclear brown signal). Scale bar: $100 \mu \mathrm{m}$. (Y) Higher-power image of boxed region in X. Arrowheads denote individual cells positive for both S100A4 and Ki67. Scale bar: $20 \mu \mathrm{m}$. (Z and AA) IHC staining of fibroblastic foci for S100A4 (red). Z is a higher power image of C. Arrow denotes thickened alveolar walls. Arrowhead denotes normal thin alveolar walls. Scale bar: 20 (Z) and 50 (AA) $\mu \mathrm{m}$. (BB) Higher-power image of $\mathbf{A}$ depicting the active fibrotic front and myofibroblast core of the polarized fibroblastic focus (100x).

D-F and $\mathrm{M}-\mathrm{O}$ ), with S100A4-expressing cells concentrated in the interface region between the focus core and relatively less involved contiguous alveolar structures (Figure 6, G, H, P, and Q).

S100A4 is not a specific marker for MPCs. Immune cells can also express S100A4 (39). Therefore, we examined fibroblastic foci at the advancing front of fibrosis for the MPC marker SSEA4. $\mathrm{SSEA}^{+}$cells codistributed with S100A4-expressing cells within the interface region of the focus (Figure 6, I, J, R, and S, and Supplemental Figure 11). To identify MPCs expressing S100A4, we performed double staining for SSEA4 and S100A4. Individual cells with dual expression of SSEA4 and S100A4 were concentrated in the interface region (Figure 6, V and W, and Supplemental Figure 12). The myofibroblast core of the focus was largely devoid of SSEA4-expressing cells. A few SSEA4 ${ }^{+}$cells were present in anatomically normal-appearing alveolar walls in control lung tissue (Supplemental Figure 13).

IPF MPCs give rise to a transit-amplifying population of daughter cells (14). Absent a definitive marker for IPF MPCs, we analyzed serial sections of fibroblastic foci for the distribution of cells that are proliferating (Ki67). Several $\mathrm{Ki} 67^{+}$cells were present in the interface region of the focus (Figure 6, K, L, T, and U). Double staining for S100A4 and Ki67 demonstrated proliferating cells $\left(\mathrm{Ki}^{+}\right)$in the interface region of the focus between Ki67-, $\alpha$-SMA ${ }^{+}$myofibroblasts in the core of the focus, and more normal alveolar structures (Figure 6X and Supplemental Figure 14). Individual cells positive for both S100A4 and Ki67 were present in the interface region, indicating that the S100A4-expressing cells in this region are actively proliferating (Figure 6Y) and define the interface region of the focus containing S100A4 ${ }^{+} / \mathrm{Ki}^{+}{ }^{+}$ expressing cells as an "active" cellular front. Importantly, we observed the presence of S100A4-expressing cells in thickened alveolar walls between normal-appearing thin alveolar walls and the region of the focus containing S100A4-expressing cells, supporting the idea that there may be an invasion of $\mathrm{S} 100 \mathrm{~A} 4^{+}$cells from the interface region into normal alveolar structures (Figure 6, $\mathrm{Z}$ and AA). These data support the concept that IPF MPCs and their early-generation progeny populate an interface region of the fibroblastic focus between the focus core and adjacent alveolar structures.

\section{Discussion}

The prevailing paradigm of IPF pathogenesis posits that epithelial injury is a key step in IPF genesis and that persistent epithelial injury activates fibroblasts, promoting fibrotic progression (40-43). Although this theory can account for disease initiation, it does not fully explain the stereotypical pattern of IPF fibrotic progression, nor does it take into account the presence of pathological MPCs as generators of fibrotic fibroblasts. We have made several recent discoveries that illuminate a path toward understanding the mechanisms of fibrotic progression. First, we identified a profibrotic feedback loop in which IPF extracellular matrix (ECM) coordinately activates expression of hundreds of connective tissue genes in lung fibroblasts by suppressing miR-29, a negative regulator of connective tissue gene translation (44). Second, we discovered fibrogenic MPCs in the IPF lung that serve as a cell of origin for the myofibroblasts mediating fibrotic destruction (14). IPF MPCs undergo robust self-renewal and manifest a durable fibrotic phenotype such that the progeny of a single MPC produces exuberant fibrotic lesions in xenograft models (14). Despite this discovery, the molecular processes underlying IPF MPC fibrogenicity and how IPF MPCs contribute to the stereotypic progression of fibrosis in IPF are not known. Here, we demonstrate that S100A4 is a key mediator underlying IPF MPC fibrogenic function and their ability to drive fibrosis.

Our data indicate that the mechanism by which S100A4 imparts fibrogenic properties to IPF MPCs involves high nuclear levels of S100A4, which functions to promote p53 degradation, stimulating IPF MPC self-renewal. While prior studies have found that S100A4 promotes proteasome-mediated degradation of $\mathrm{p} 53$ protein, the precise mechanism remains unclear. One study found that S100A4 binds p53 directly (25), while other work suggests that S100A4 indirectly interacts with p53 to stimulate its degradation (26-28). Our analytical studies using mass spectrometry to define the IPF MPC S100A4 nuclear interactome reveal that S100A4 interacts with PIMT and members of the proteasome complex within the nucleus. By methylating p53, PIMT enhances p53 interaction with HDM2, promoting its degradation (34). Our work indicates that the nuclear S100A4 promotes 53 proteasomal degradation by complexing with PIMT. We found that PIMT depletion, even in the context of excess S100A4, enhanced p53 levels, demonstrating the pivotal role PIMT plays in the ability of S100A4 to promote p53 degradation. These data indicate that the S100A4 nuclear complex containing PIMT and members of the proteasome degrade p53, stimulating IPF MPC proliferation and expansion of the IPF MPC population. It is important to note that, depending on its location in the cell, S100A4 may be capable of regulating other cellular processes that are crucial to fibrotic progression. Therefore, it will be important to perform additional studies to examine: i) the cellular location of S100A4 as IPF MPCs differentiate to IPF fibroblasts; ii) the function of cytoplasmic S100A4 in IPF cells and whether it regulates motility/invasion; and iii) whether S100A4 is secreted by IPF MPCs and/or their progeny, thereby influencing the biology of 
neighboring cells. While important, these studies are beyond the scope of the current body of work.

S100A4 has also been termed metastasin because it regulates cancer cell metastasis (15-22). While S100A4 levels are elevated in both cancer cells and IPF cells, IPF cells do not metastasize, suggesting that there are important differences between cancer and fibrosis in relation to S100A4 function. Metastasis is a complicated biological process involving cellular transformation, angiogenesis, motility and invasion, transport to distant sites, and complicated tumor-host relationships (45). Thus, while S100A4 can mediate processes involved in cancer metastasis, such as motility/ invasion, there are other molecular processes required for a cancer cell to be able to metastasize that do not involve S100A4. Furthermore, S100A4 functions by interacting with other proteins. It is likely that the cancer cell S100A4 interactome is different than the IPF cell S100A4 interactome, resulting in S100A4 mediating different functions. Thus, in cancer cells, S100A4 in concert with other molecular processes facilitates metastasis, while in IPF, high levels of S100A4 by itself does not confer a metastatic phenotype.

The lack of therapies that stop IPF fibrotic progression stems in part from the absence of in vitro or in vivo models that recapitulates the stereotypical anatomy of IPF fibrotic progression (46). Our existing models create self-limited lung fibrosis but are not progressive and do not recapitulate the development of multifocal heterogeneous fibroblastic foci, which is the diagnostic feature of IPF $(9,10)$. We found that human IPF MPCs can convert the self-limited bleomycin mouse model of lung fibrosis to a model of persistent fibrosis in an S100A4-dependent manner. We have also found that overexpression of S100A4 confers control MPCs with fibrogenic properties. However, it is important to note that there were differences in the fibrotic response of mice pretreated with bleomycin that received IPF MPCs and those that received control MPCs overexpressing S100A4. The collagen content was lower in the lungs of mice receiving control MPCs overexpressing S100A4 compared with the lungs of mice receiving unaltered IPF MPCs (288 $\pm 18 \mu \mathrm{g} /$ left lung vs. $846 \pm 151 \mu \mathrm{g} /$ left lung), and the histology of the lungs of mice receiving control MPCs overexpressing S100A4 did not show the extensive intraparenchymal fibrotic lesions infiltrated with procollagen-expressing human cells and S100A4-expressing cells that were common in the lungs of mice receiving IPF MPCs. This suggests that IPF MPCs are more fibrogenic than control MPCs overexpressing S100A4. In our prior publication (14), our genomewide transcriptomic analysis revealed multiple gene-expression differences between control and IPF MPCs. This result suggests that, in addition to alterations in S100A4 expression, other gene-expression differences are likely responsible for conferring IPF MPCs with their entire pathologic phenotype. Together, our results indicate that S100A4 is necessary to confer IPF MPCs with fibrogenicity and sufficient to confer control MPCs with fibrotic properties. This represents a major advance in the field, as it is the first mammalian xenograft model of persistent lung fibrosis using human IPF lung MPCs. In this model, the increased fibrosis results from expansion of fibrogenic human mesenchymal cells within the mouse lung and their subsequent deposition of human ECM. Our results have several major implications. First, S100A4 antagonism, which is being exploited for cancer treatment, can be considered a promising approach to disrupt IPF MPC function and arrest IPF fibrotic progression. Second, the incorporation of human IPF MPCs into the bleomycin mouse model creates a model of persistent fibrosis that can be employed as a personalized mouse model, where implantation of patient MPCs in mice can be tested in drug-efficacy studies.

IPF progresses in a stereotypic manner. The disease begins in the peripheral basilar portions of the lung. As IPF progresses, the fibrosis expands centrally. However, the mechanisms that underlie this nonrandom progression of fibrosis in IPF have remained elusive. Recent work defining the morphologic structure of fibroblastic foci indicates that they are heterogeneous structures that vary greatly in size, raising the possibility that there is temporal growth of fibroblastic foci as the fibrotic lesions evolve by spread of fibrosis into adjacent normal alveolar structures (10). Our immunohistochemical studies examining fibroblastic foci support this concept and may provide a cogent explanation for this stereotypic progression of fibrosis. We demonstrate that fibroblastic foci, located at the advancing front of fibrosis, are polarized structures with $\alpha-\mathrm{SMA}^{+}$myofibroblasts in the focus core and SSEA $4^{+}$/ S100A4 ${ }^{+} \mathrm{MPCs}^{-}$and SSEA4 $4^{-} / \mathrm{S} 100 \mathrm{~A} 4^{+}$-expressing cells located in the focus periphery at the interface between the myofibroblastic core and adjacent intact alveolar structures. S100A4-expressing cells populating this interface are proliferating $\left(\mathrm{Ki}^{+} 7^{+}\right)$, suggesting that this region represents an active fibrotic front. Importantly, we found that S100A4 ${ }^{+}$cells are present in thickened alveolar walls juxtaposed between the active cellular front of the focus and morphologically intact alveolar walls, consistent with the idea that S100A4-expressing cells spread the disease into immediate adjacent uninvolved alveolar structures. Our IHC studies show that $\alpha-\mathrm{SMA}^{+}$myofibroblasts in the focus core lack S100A4 expression. Our in vitro data indicate that, as IPF MPCs differentiate to myofibroblasts, S100A4 expression decreases. This suggests that, as the fibrotic front advances, the cells behind the active front, which were formerly IPF MPCs, differentiate to $\alpha-\mathrm{SMA}^{+} / \mathrm{S} 100 \mathrm{~A} 4^{-}$ myofibroblasts that constitute the focus core. These data support the concept that the IPF fibroblastic focus is a polarized structure consisting of an active fibrotic front containing MPCs (SSEA4 ${ }^{+}$/ S100A4 $4^{+}$and early-generation MPC-derived transit amplifying daughter cells $\left(\mathrm{SSEA} 4^{-} / \mathrm{S} 100 \mathrm{~A} 4^{+} / \mathrm{Ki}^{+}\right)$and a core composed of myofibroblasts $\left(\alpha-\mathrm{SMA}^{+} /\right.$procollagen $^{+}$; Figure $\left.6 \mathrm{BB}\right)$.

The presence of fibrogenic MPCs, fibroblasts with a durable fibrogenic phenotype, and an ECM that suppresses expression of antifibrotic microRNAs are key elements of IPF fibrotic progression that are not accounted for in existing models of lung fibrosis. On the basis of our published and current data, we propose a paradigm that incorporates these missing elements and offers a cogent explanation for the stereotypic progression of fibrosis in IPF. The fibroblastic focus can be conceptualized as a fibrogenic niche that harbors and nurtures IPF MPCs and their progeny. The focus is a polarized structure with an active fibrotic front containing selfrenewing MPCs and a transit-amplifying population of MPC progeny residing in an ECM that supports their pathological properties. The fibrotic front infiltrates into adjacent normal alveolar walls coursing in an axial distribution to mediate fibrotic lung destruction. We suggest that targeting S100A4-expressing IPF MPCs, the actual cells comprising the active fibrotic front of the fibroblastic focus, may be an effective therapeutic strategy to arrest IPF fibrotic progression. 


\section{Methods}

Primary mesenchymal cell lines. Seven primary lung mesenchymal cell lines were established from patients fulfilling diagnostic criteria for IPF, including a pathological diagnosis of usual interstitial pneumonia (47). Patient controls were selected to be similar in age to IPF patients with nonfibrotic lung disorders. Based on these criteria, we utilized 7 nonfibrotic primary control fibroblast lines from lung tissue uninvolved by the primary disease process: adenocarcinoma $(n=3)$, squamous cell carcinoma $(n=3)$, or histologically normal lung tissue from a gunshot victim $(n=1)$. Cell lines were derived from lungs, characterized as mesenchymal cells, and cultivated as previously described (14).

Isolation of MPCs. IPF and control MPCs were isolated from primary IPF and control mesenchymal cell cultures at passage 0 (initial isolate before subcultivation) through passage 4 . To isolate progenitors, primary IPF and control mesenchymal cells were labeled with mouse anti-human SSEA4 antibody conjugated to Alexa Fluor 647 (clone MC-813-70, catalog 560796, BD Biosciences) and mouse anti-human SSEA1 conjugated to PE (clone MC480, catalog 560142, BD Biosciences) as previously described (14). Cells were sorted on a FACSAria Cell Sorter (BD Biosciences). Cells that were SSEA4 ${ }^{+}$and SSEA1- (relative to mouse IgG3 $\kappa$ isotype control conjugated to Alexa Fluor 647 and mouse IgM $\kappa$ isotype control conjugated to PE, respectively; clone J606, catalog 560803; clone G155-228, catalog 555584; BD Biosciences) and $<12 \mu \mathrm{m}$ were collected. To generate sufficient numbers of MPCs for the in vivo mouse studies, the SSEA4 ${ }^{+}$MPCs were expanded by culture in DMEM $+10 \% \mathrm{FCS}$ for 7 days prior to use. The resulting MPC cultures were analyzed for SSEA4 expression by FACs analysis and for colony formation in vitro. Day 7 MPCs (97\%) were SSEA4 ${ }^{+}$, and the cells formed colonies in methylcellulose, indicating retention of progenitor self-renewal properties.

Quantitative PCR. Validity testing of S100A4 gene expression was conducted by quantitative PCR (qPCR) as previously described (14). Total RNA was isolated and reverse transcribed using a Taqman Reverse Transcriptase Reagent Kit (Roche Diagnostics) and primed with random hexamers. Primer sequences were selected using NCBI Primer-BLAST. qPCR was performed using a LightCycler FastStart DNA Master ${ }^{\text {PLus }}$ SYBR Green I Kit (Roche Diagnostics). Primer sequences were as follows: S10OA4 forward, $5^{\prime}$ GTACGTGTTGATCCTGACTGCTGTCATGG 3'; S100A4 reverse, 5' TCATTTCTTCCTGGGCTGCTTATCTGGG $3^{\prime}$. Samples were quantified at the log-linear portion of the curve using LightCycler analysis software and compared with an external calibration standard curve.

Self-renewal assay. Single cell suspensions of SSEA4 $4^{+}$IPF MPCs were incorporated into methylcellulose gels (STEMCELL Technologies) and maintained in MSC SFM CTS (Thermo Scientific/Gibco; $37^{\circ} \mathrm{C}, 5 \% \mathrm{CO}_{2}$; 1 week). Enumeration of colonies was performed microscopically.

Plasmids/constructs. For gain of S100A4 function, we overexpressed S100A4 using a WT S100A4 construct (S100A4-GFP-tagged construct RG203035, Origene) in a lentiviral vector. Empty vector served as a control. For loss of S100A4 function, S100A4 was knocked down using S100A4 shRNA (pGIPZS100A4 shRNA, Addgene). Scrambled shRNA served as a control. For gain of p53 function, we overexpressed p53 using a WT p53 construct in an adenoviral vector (Ad-p53-GFP, catalog 1260, Vector Biolabs). Empty vector served as a control. For loss of PIMT function, PIMT was knocked down using PIMT shRNA (catalog sc-45875, Santa Cruz Biotechnology Inc.). Scrambled shRNA served as a control.
Mass spectrometry. Nuclear fractions of IPF MPCs were isolated by NE-PER Nuclear and Cytoplasmic Extraction reagents (Thermo Scientific). S100A4 was immunoprecipitated from the resulting nuclear lysates using an S100A4 antibody (catalog sc-377059, Santa Cruz Biotechnology Inc.). Control consisted of immunoprecipitation using an isotype control antibody. The immunoprecipitates were subjected to SDS-PAGE followed by silver staining (Pierce Silver Stain Kit, catalog 24612, Thermo Fisher Scientific). Bands were excised, destained, and subjected to in-gel trypsin digestion. Peptide samples were resuspended in 98:2 water/acetonitrile, $0.1 \%$ formic acid and run on an Orbitrap Velos (Thermo Fisher Scientific) mass spectrometer. The Thermo RAW files were analyzed with Peaks Studio 7.0 build 20140912 (Bioinformatics Solutions) software package for interpretation of tandem mass spectometry and protein inference.

Western blot and immunoprecipitation. Blots were performed as previously described (11-13). For immunoprecipitation, nuclear fractions were isolated by NE-PER Nuclear and Cytoplasmic Extraction reagents. The samples were centrifuged at $120,000 \mathrm{~g}$ for 15 minutes at $4^{\circ} \mathrm{C}$, and the lysates were precleared for 1 hour at $4^{\circ} \mathrm{C}$ with protein $\mathrm{A} / \mathrm{G}$ beads and immunoprecipitated for 2 hours at $4^{\circ} \mathrm{C}$ with the appropriate primary antibody. The samples were processed for Western blot analysis.

IHC. IHC was performed on $4-\mu \mathrm{m}$, paraffin-embedded, serialsectioned IPF and control lung tissue and mounted on polylysine-coated slides. The sections were deparaffinized in xylene, rehydrated through a graded methanol series, quenched with $0.3 \%$ hydrogen peroxide in methanol, and immersed in a $98^{\circ} \mathrm{C}$ water bath for 30 minutes in citrate buffer ( $\mathrm{pH}$ 6.0) for antigen retrieval. Sections were placed in $5 \%$ Normal Horse Serum (Jackson Immunoresearch Laboratories) to block nonspecific binding of secondary antibodies. A multiplex IHC kit was used for antigen detection according to the manufacturer's instructions (MULTIVIEW IHC Kit, product no. ADI-950-101-0001, Enzo Life Sciences). The tissue specimens were incubated overnight (18-20 hours, $\left.4^{\circ} \mathrm{C}\right)$ in the monoclonal human primary antibodies; $\alpha$-SMA (1:2,500, catalog A2547, Sigma-Aldrich), S100A4 (1:2,000, catalog sc-377059, Santa Cruz Biotechnology Inc.), and Ki67 (1:400, catalog MA5-14520, Thermo Scientific). SSEA4 IHC was performed using mouse anti-human SSEA4 antibody (1:100, clone MC-813-70, catalog 330402, BioLegend). Sections were counterstained with Hematoxylin (Invitrogen) for 2 minutes, and PBS was applied for 30 minutes. Specimens were cover-slipped with a Prolong Antifade Kit (Invitrogen, Molecular Probes) and stored overnight at room temperature without light before image analysis.

Mouse model of persistent fibrosis. To assess the role of IPF MPCs in fibrotic progression, we utilized the bleomycin model of lung fibrosis to determine whether IPF MPCs could convert the bleomycin model from a self-limited regressing model to a model of persistent lung fibrosis. NOD/SCID/IL2ry/B2M (NSG) male and female mice (Jackson Laboratories), average 10 weeks of age, were administered intratracheal bleomycin $(1.25 \mathrm{U} / \mathrm{kg})$ to establish self-limited lung fibrosis. Mice treated with intratracheal saline were used as controls. The lungs were harvested 6 weeks after intratracheal bleomycin (or saline) administration. To test whether IPF MPCs can drive fibrotic progression, 2 weeks following bleomycin administration, IPF or control MPCs were suspended in PBS (1 $\times 10^{6}$ cells $\left./ 100 \mu \mathrm{l}\right)$ and injected into the mice via the tail vein following our published protocol (14). Mice were euthanized 4 weeks after adoptive transfer of human cells. For the S100A4-knockdown studies, mice were treated with intratracheal bleomycin. Two weeks later, IPF MPCs infected with a lentiviral vector containing S100A4 shRNA were admin- 
istered by tail vein injection. IPF MPCs containing scrambled shRNA served as controls. Engraftment of human IPF MPCs treated with S100A4 shRNA and scrambled shRNA in the immune-deficient mice were confirmed using a previously published real-time PCR protocol that has been shown to be sensitive for the detection and quantitation of human cells in mice (37). The lungs were harvested 4 weeks after cell administration. Collagen content was quantified in the left-lung tissue by Sircol assay and served as the primary endpoint (48). Histological (H\&E and trichrome staining) and immunohistochemical analyses were performed on paraffin-embedded and frozen right-lung tissue. Cells positive for human procollagen (anti-human procollagen type I antibody, 1:500, catalog ab64409; Abcam) were identified as human. The presence of lung fibrotic lesions by histological analysis served as the secondary endpoint. IHC using an S100A4 antibody was performed to assess the distribution of S100A4-expressing cells with human procollagen I-expressing cells.

RNA sequencing data. We used previously generated RNA sequencing data (14). The complete datasets and protocols are deposited in data repository Gene Expression Omnibus (GEO; accession number GSE97038).

Statistics. Comparisons of data among experiments were performed using the 2 -ailed Student's $t$ test. Experiments were independently replicated a minimum of 3 times. Data are expressed as mean \pm SEM. $P<0.05$ was considered significant.

Study approval. Deidentified patient samples were obtained under a waiver of informed consent from the University of Minnesota IRB. Animal protocols were approved and conducted in accordance with the University of Minnesota Institutional Animal Care and Use Committee regulations.

\section{Author contributions}

$\mathrm{CAH}$ conceived, designed, and directed the studies, with input from $\mathrm{HX}$ and $\mathrm{PBB}$. CAH and $\mathrm{PBB}$ wrote the manuscript, with assistance from all authors. HX established primary human mesenchymal cell lines, cultured MPCs, performed flow cytometry for isolation of MPCs, performed qPCR and Western blot analysis, assisted with mass spectrometry experiments, and performed gain- and loss-offunction experiments, mouse studies, and IHC. AG assisted with isolation and culture of MPCs, assisted with mass spectrometry experiments, and assisted with mouse studies. $\mathrm{AB}$ assisted with flow cytometry. JH established primary human mesenchymal cell lines and performed immunohistochemical analysis. ER acquired human tissue samples and performed histological and immunohistochemical analysis of lung tissue specimens. KS designed and constructed expression constructs and performed flow cytometry for isolation of MPCs. MP established primary human mesenchymal cell lines, assisted in RNA sequencing experiments, and assisted in flow cytometry for isolation of MPCs and zebrafish work. TG designed and analyzed mass spectrometry experiments. LY established primary human mesenchymal cell lines, performed cell culturing, and assisted in flow cytometry for isolation of MPCs.

\section{Acknowledgments}

We would like to acknowledge the assistance of the Flow Cytometry Core Facility of the Masonic Cancer Center, a comprehensive cancer center designated by the National Cancer Institute, supported in part by P30 CA77598 and the University of Minnesota Imaging Center. We also acknowledge the Center for Mass Spectrometry and Proteomics. This work was supported by NIH grants R01 HL125227 to CAH and R01 HL125236 to PBB, as well as by funds provided by the O'Brien and Witowski families.

Address correspondence to: Craig A. Henke, Box 276, University of Minnesota, 420 Delaware Street SE, Minneapolis, Minnesota 55455, USA. Phone: 612.624.0999; Fax: Email: henke002@umn.edu.
1. Raghu G, Weycker D, Edelsberg J, Bradford WZ, Oster G. Incidence and prevalence of idiopathic pulmonary fibrosis. Am J Respir Crit Care Med. 2006;174(7):810-816.

2. Noble PW. Idiopathic pulmonary fibrosis: natural history and prognosis. Clin Chest Med. 2006;27(1 Suppl 1):S11-S16.

3. Kim DS, Collard HR, King TE. Classification and natural history of the idiopathic interstitial pneumonias. Proc Am Thorac Soc. 2006;3(4):285-292.

4. Kuhn C, McDonald JA. The roles of the myofibroblast in idiopathic pulmonary fibrosis. Ultrastructural and immunohistochemical features of sites of active extracellular matrix synthesis. Am J Pathol. 1991;138(5):1257-1265.

5. Nishimura K, Kitaichi M, Izumi T, Nagai S, Kanaoka M, Itoh $\mathrm{H}$. Usual interstitial pneumonia: histologic correlation with high-resolution CT. Radiology. 1992;182(2):337-342.

6. Katzenstein AL, Myers JL. Idiopathic pulmonary fibrosis: clinical relevance of pathologic classification. Am J Respir Crit Care Med. 1998;157(4 Pt 1):1301-1315.

7. Wynn TA, Ramalingam TR. Mechanisms of fibrosis: therapeutic translation for fibrotic disease. Nat Med. 2012;18(7):1028-1040.

8. Noble PW, Barkauskas CE, Jiang D. Pulmonary fibrosis: patterns and perpetrators. JClin Invest.
2012;122(8):2756-2762.

9. Cool CD, Groshong SD, Rai PR, Henson PM, Stewart JS, Brown KK. Fibroblastic foci are not discrete sites of lung injury or repair: the fibroblast reticulum. Am JRespir Crit Care Med. 2006;174(6): 654-658.

10. Jones MG, et al. Three-dimensional characterization of fibroblast foci in idiopathic pulmonary fibrosis. JCI Insight. 2016;1(5): e86375.

11. Xia H, et al. Pathological integrin signaling enhances proliferation of primary lung fibroblasts from patients with idiopathic pulmonary fibrosis. J Exp Med. 2008;205(7):1659-1672.

12. Xia H, Khalil W, Kahm J, Jessurun J, Kleidon J, Henke CA. Pathologic caveolin-1 regulation of PTEN in idiopathic pulmonary fibrosis. Am J Pathol. 2010;176(6):2626-2637.

13. Xia H, et al. Low $\alpha(2) \beta(1)$ integrin function enhances the proliferation of fibroblasts from patients with idiopathic pulmonary fibrosis by activation of the $\beta$-catenin pathway. Am J Pathol. 2012;181(1):222-233.

14. Xia H, et al. Identification of a cell-of-origin for fibroblasts comprising the fibrotic reticulum in idiopathic pulmonary fibrosis. Am J Pathol. 2014;184(5):1369-1383.

15. Garrett SC, Varney KM, Weber DJ, Bresnick AR. S100A4, a mediator of metastasis. J Biol Chem.
2006;281(2):677-680.

16. Lo JF, et al. The epithelial-mesenchymal transition mediator S100A4 maintains cancer-initiating cells in head and neck cancers. Cancer Res. 2011;71(5):1912-1923.

17. Yan XL, et al. Hepatocellular carcinoma-associated mesenchymal stem cells promote hepatocarcinoma progression: role of the S100A4-miR155-SOCS1-MMP9 axis. Hepatology. 2013;57(6):2274-2286.

18. Harris MA, et al. Cancer stem cells are enriched in the side population cells in a mouse model of glioma. Cancer Res. 2008;68(24):10051-10059.

19. Kanojia D, et al. Proteomic profiling of cancer stem cells derived from primary tumors of HER2/Neu transgenic mice. Proteomics. 2012;12(22):3407-3415.

20. Chen X, et al. The E-F hand calcium-binding protein S100A4 regulates the proliferation, survival and differentiation potential of human osteosarcoma cells. Cell Physiol Biochem . 2013;32(4):1083-1096.

21. Eisenbacher JL, et al. S100A4 and uric acid promote mesenchymal stromal cell induction of IL-10+/IDO+ lymphocytes. J Immunol. 2014;192(12):6102-6110.

22. Schmidt-Hansen B, et al. Functional significance of metastasis-inducing S100A4(Mts1) 
in tumor-stroma interplay. J Biol Chem. 2004;279(23):24498-24504.

23. Tomcik M, et al. S100A4 amplifies TGF- $\beta$ induced fibroblast activation in systemic sclerosis. Ann Rheum Dis. 2015;74(9):1748-1755.

24. Donato R, et al. Functions of S100 proteins. Curr Mol Med. 2013;13(1):24-57.

25. Grigorian M, et al. Tumor suppressor p53 protein is a new target for the metastasisassociated Mts1/S100A4 protein: functional consequences of their interaction. J Biol Chem. 2001;276(25):22699-22708.

26. Orre LM, et al. S100A4 interacts with p53 in the nucleus and promotes $\mathrm{p} 53$ degradation. Oncogene. 2013;32(49):5531-5540.

27. Fernandez-Fernandez MR, Veprintsev DB, Fersht AR. Proteins of the $\mathrm{S} 100$ family regulate the oligomerization of $\mathrm{p} 53$ tumor suppressor. Proc Natl Acad Sci USA. 2005;102(13):4735-4740.

28. van Dieck J, Lum JK, Teufel DP, Fersht AR. S100 proteins interact with the $\mathrm{N}$-terminal domain of MDM2. FEBS Lett. 2010;584(15):3269-3274.

29. Tamaki Y, et al. Metastasis-associated protein, S100A4 mediates cardiac fibrosis potentially through the modulation of $\mathrm{p} 53$ in cardiac fibroblasts. J Mol Cell Cardiol. 2013;57:72-81.

30. Li ZH, Bresnick AR. The S100A4 metastasis factor regulates cellular motility via a direct interaction with myosin-IIA. Cancer Res. 2006;66(10):5173-5180.

31. Dahlmann M, et al. RAGE mediates S100A4induced cell motility via MAPK/ERK and hypoxia signaling and is a prognostic biomarker for human colorectal cancer metastasis. Oncotarget. 2014;5(10):3220-3233.
32. Pistoia V, Raffaghello L. Damage-associated molecular patterns (DAMPs) and mesenchymal stem cells: a matter of attraction and excitement. Eur J Immunol. 2011;41(7):1828-1831.

33. Ellson CD, Dunmore R, Hogaboam CM, Sleeman MA, Murray LA. Danger-associated molecular patterns and danger signals in idiopathic pulmonary fibrosis. Am J Respir Cell Mol Biol. 2014;51(2):163-168.

34. Lee JC, et al. Protein L-isoaspartyl methyltransferase regulates 533 activity. Nat Commun. 2012;3:927.

35. Zhang K, Rekhter MD, Gordon D, Phan SH. Myofibroblasts and their role in lung collagen gene expression during pulmonary fibrosis. A combined immunohistochemical and in situ hybridization study. Am J Pathol. 1994;145(1):114-125.

36. Izbicki G, Segel MJ, Christensen TG, Conner MW, Breuer R. Time course of bleomycin-induced lung fibrosis. Int J Exp Pathol. 2002;83(3):111-119.

37. Lawson WE, et al. Characterization of fibroblast-specific protein 1 in pulmonary fibrosis. Am J Respir Crit Care Med. 2005;171(8):899-907.

38. Song P, Xie Z, Guo L, Wang C, Xie W, Wu Y. Human genome-specific real-time PCR method for sensitive detection and reproducible quantitation of human cells in mice. Stem Cell Rev. 2012;8(4):1155-1162.

39. Taylor S, Herrington S, Prime W, Rudland PS, Barraclough R. S100A4 (p9Ka) protein in colon carcinoma and liver metastases: association with carcinoma cells and T-lymphocytes. Br JCancer. 2002;86(3):409-416.

40. Fernandez IE, Eickelberg O. New cellular and molecular mechanisms of lung injury and fibrosis in idiopathic pulmonary fibrosis. Lancet.
2012;380(9842):680-688.

41. King TE, Pardo A, Selman M. Idiopathic pulmonary fibrosis. Lancet. 2011;378(9807):1949-1961.

42. Noble PW, Homer RJ. Idiopathic pulmonary fibrosis: new insights into pathogenesis. Clin Chest Med. 2004;25(4):749-758, vii.

43. Selman M, King TE, Pardo A, American Thoracic Society, European Respiratory Society, American College of Chest Physicians. Idiopathic pulmonary fibrosis: prevailing and evolving hypotheses about its pathogenesis and implications for therapy. Ann Intern Med 2001;134(2):136-151

44. Parker MW, et al. Fibrotic extracellular matrix activates a profibrotic positive feedback loop. J Clin Invest. 2014;124(4):1622-1635.

45. Boye K, Maelandsmo GM. S100A4 and metastasis: a small actor playing many roles. Am J Pathol. 2010;176(2):528-535.

46. Moeller A, Ask K, Warburton D, Gauldie J, Kolb M. The bleomycin animal model: a useful tool to investigate treatment options for idiopathic pulmonary fibrosis? Int J Biochem Cell Biol. 2008;40(3):362-382.

47. [No authors listed]. American Thoracic Society. Idiopathic pulmonary fibrosis: diagnosis and treatment. International consensus statement. American Thoracic Society (ATS), and the European Respiratory Society (ERS). Am J Respir Crit Care Med. 2000;161(2 Pt 1):646-664.

48. Pierce EM, et al. Therapeutic targeting of CC ligand 21 or CC chemokine receptor 7 abrogates pulmonary fibrosis induced by the adoptive transfer of human pulmonary fibroblasts to immunodeficient mice. Am JPathol. 2007;170(4):1152-1164. 